lich mehr als unter Rentnern“. Auch beurteilten deutlich mehr Deutsche als deutschsprachige Ausländer die Diskretion am Praxisempfang als wenig vertraulich. Am schlechtesten wird sie von Frauen bewertet: $20 \%$ der Umfrageteilnehmerinnen zwischen 18 und 59 Jahren entschieden sich für die vorgegebene Negativkategorie ,überhaupt nicht gut“. KBV-Vize Regina Feldmann kündigte an, dass die KBV auf diesen Aspekt der Umfrage reagieren werde: „Wenn die Hälfte der Befragten den Schutz der persönlichen Belange als unzureichend empfindet, dann muss uns das für die Praxisorganisation zu denken geben.“

Für die mitunter langen Wartezeiten auf Termine ernten die Vertragsärzte dagegen wenig Kritik. $81 \%$ der mehr als 6.000 Befragten stören sich nicht daran. Auch diese Werte sind seit den Anfängen der Versichertenbefragung stabil. Uner- freulich: Immerhin $8 \%$ der Patienten, die sich im Vorjahr mit einem akuten Problem beim Arzt gemeldet hatten, mussten auf einen Termin mehr als drei Wochen lang warten. Klare Unterschiede gibt es zwischen Haus- und Fachärzten: $73 \%$ der Akutpatienten in den Hausarztpraxen mussten gar nicht warten. In den Facharztpraxen dreht sich das Verhältnis um: 70\% der Akutpatienten mussten mindestens einen Tag warten, $55 \%$ sogar mehr als drei Tage.

Die KBV-Umfrage fand 2016 zum achten Mal statt. Erhebungszeitraum war Mitte März bis Anfang Mai. Die erste Befragung dieser Art ist datiert auf 2006. An der diesjährigen Telefonumfrage beteiligten sich 6.113 Bundesbürger. Der Berichtsband mit allen Umfrageergebnissen gibt es als PDF-Datei auf der KBV-Website http://bitly/2bQmD8P.

Anno Fricke und Christoph Winnat

\title{
Fallzahl als Stellgröße stets im Blick behalten
}

\author{
Die Honorarverteilungsmaßstäbe der KVen sind für Ärzte oft ein Buch mit \\ sieben Siegeln. Ein Wert, auf den geschaut werden sollte: die Fallzahl.
}

$\mathrm{D}$ ie Abrechnungsbescheide der KVen sind für Ärzte ein wichtiges Hilfsmittel, um festzustellen, wo sie mit ihrer Praxis betriebswirtschaftlich stehen. Vor allem bei den Größen, wo es einen Fachgruppenvergleich gibt, lässt sich der Bescheid gut für eine Art Benchmarking nutzen. Doch schrecken viele Ärzte vor dem Zahlenwust der Abrechnungsbescheide zurück, zumal die Verteilungssystematik der Honorare in der KV, die über den Honorarverteilungsmaßstab festgelegt wird, vielen nur schwer verständlich ist, weil jede KV hier andere Regeln hat. Einige Leitplanken bei der Analyse der Abrechnungsbescheide gibt es aber doch: „Eine wichtige Einflussgröße auf das Budget ist auf jeden Fall die Fallzahl pro Quartal. Sie sollte stets im Blick bleiben“, empfiehlt Georg Heßbrügge, Bereichsleiter Gesundheitsmärkte- und -politik bei der Deutschen Apotheker- und Ärztebank (apoBank). Die Fallzahl im aktuell laufenden Quartal habe direkte Auswirkungen auf das Budget im Quartal des Folgejahres - je- denfalls in KVen, die mit Regelleistungsvolumen und qualitätsgebundenen $\mathrm{Zu}$ satzvolumen arbeiten. Damit beeinflusse sie die potenziellen zukünftigen Einnahmen der Praxis. „Die Entwicklung der Fallzahl kann somit auch als Indikator für sich abzeichnende finanzielle Veränderungen in der Arztpraxis dienen“, so der Hinweis Heßbrügges.

\section{Budget ausschöpfen, aber nicht überschreiten}

In großen Versorgerpraxen, die meist stark frequentiert sind, werden kleine Änderungen bei der Fallzahl nicht so sehr ins Gewicht fallen, weil diese Praxen ohnehin für Werte oberhalb einer bestimmten Fallzahl in den meisten KVen nur abgestaffelt vergütet werden. In kleineren Praxen können sich geringere Fallzahlen direkt auf das Einkommen auswirken. Heßbrügge empfiehlt daher, das individuelle, von der $\mathrm{KV} z u-$ gewiesene Budget auszuschöpfen und eine zu hohe Budgetüberschreitung $\mathrm{zu}$ vermeiden.

Hauke Gerlof

\section{MRSA: Leistungsbedarf in Praxen steigt leicht an}

Seit Inkrafttreten der MRSA-Vergütungsvereinbarung im Quartal II/2012 ist die Anzahl der Vertragsärzte, die Leistungen zur Diagnostik und Therapie von Patienten mit (Verdacht auf) Befall durch methicillinresistenten Staphylokokkus aureus (MRSA) erbringen, kontinuierlich gestiegen. Das zeigt der KBV-Honorarbericht für das Jahr 2014. Demnach rechneten im Quartal II/2014 bereits 5.148 Vertragsärzte MRSA-Leistungen ab, $10 \%$ mehr als zwei Jahre zuvor, als die neuen Leistungspositionen eingeführt wurden. Damit nutzte knapp jeder dritte Arzt mit Genehmigung die betreffenden EBMZiffern: 16.273 Vertragsärzte hatten zum Stichtag 31. März 2014 eine MRSA-Abrechnungsgenehmigung. Es geht um die Leistungen des Kapitels 30.12 im EBM, "Spezielle Diagnostik und Eradikationstherapie im Rahmen von MRSA", das selbst zwar erst im Quartal II/2014 eingeführt wurde. Die Leistungen konnten jedoch aufgrund der Vergütungsvereinbarungen als 86er-Ziffern bereits vorher abgerechnet werden.

Der Honorarbericht vermittelt erstmals einen Einblick, wie hoch der Bedarf für die neuen Leistungen tatsächlich ist. Nach den Daten des KBV-Honorarberichts hat im Zeitraum vom Quartal II/2012 bis Quartal II/2014 die Zahl der untersuchten und behandelten Risikopatienten von 11.368 auf 12.551 Patienten leicht zugenommen (+10,4\%). Blickt man auf die Anzahl der Eradikationstherapien bei MRSA-Infektion (EBM-Ziffer 30942), so zeigt sich ein leichter Rückgang von 3,7\% auf 5.601 Eradikationstherapien.

Hauke Gerlof

\section{Schmerzmedizin: Zugang für Ärzte wird erleichtert}

Der Zugang zur Versorgung chronisch schmerzkranker Patienten wird für Vertragsärzte erleichtert. Das sieht die erneuerte Schmerztherapie-Vereinbarung vor, auf die sich KBV und GKV-Spitzenverband geeinigt haben. Damit werden die Anforderungen an die aktuellen Vorgaben der Zusatzweiterbildung "Spezielle Schmerztherapie" angepasst, heißt es. Auch die Praxisbedingungen würden nun flexibler gestaltet. Die Modalitäten zur Dokumentationsprüfung wurden verändert.

Weitere Informationen bei der KBV: www. kbv.de/media/sp/Schmerztherapie.pdf Hauke Gerlof 